
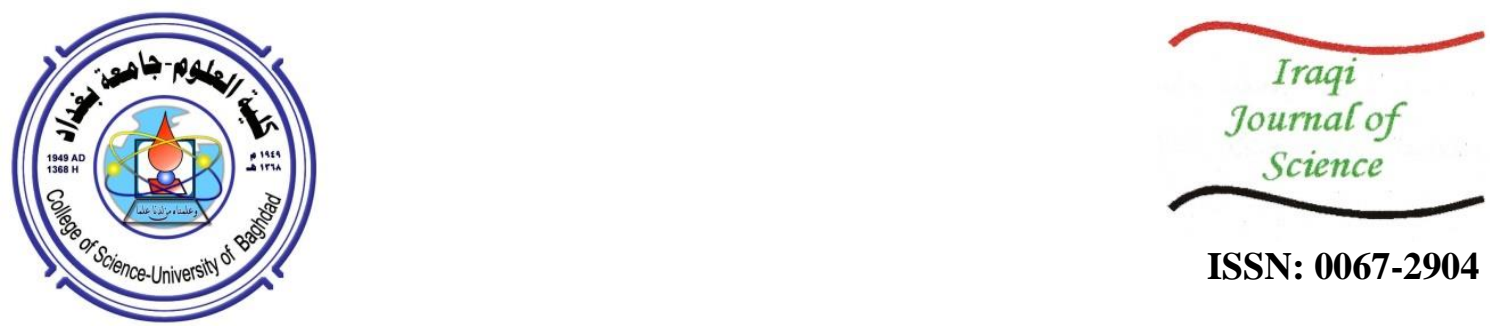

ISSN: 0067-2904

\title{
Relation between Coronal Mass Ejections and Sunspot Number during Solar Cycle 24
}

\author{
Zeinab F. Hussein \\ Department of Space and Astronomy, College of Science, University of Baghdad, Baghdad,Iraq
}

\begin{abstract}
In this study, we report a statistical study for the relationship between coronal mass ejections (CMEs) and sunspot number (SSN) that were registered during the period 2008-2017 for the solar cycle 24. SSN was extracted from Sunspot Index and Long-term Solar Observations (SILSO), while CMEs number from observations made by the Large Angle and Spectrometric Coronagraph (LASCO) on board the Solar and Heliospheric Observatory mission (SOHO). The present period was adopted to conduct the investigation and obtain the mutual correlation between SSN and CMEs. The relationship between CME, the speed of halo CME, and partial halo CMEs for solar cycle 24 were studied. The analysis of results indicated that the average speed of halo CMEs is almost faster than the average speed of partial halo CMEs.Test results of the annual correlation between SSN and CMEs are simple and can be represented by a linear regression equation. Finally, Gaussian fit as a function of time was performed to compare behavior of numbers the CME and SSN with the years and the results show that the center of the peaks agrees with 2014.
\end{abstract}

Keywords: Coronal mass ejection, Sunspot number, halo CMEs, partial halo CMEs and solar cycle.

\section{العلاقة بين المقذوفات الكتلية ألأكليلية الثمسية وعدد البقع الثمسية خلال الدورة الثمسية 24}

$$
\begin{aligned}
& \text { زينب فاضل حسين } \\
& \text { قسم الفلك والفضاء ، كلية العلوم ، جامعة بغداد ، بغداد ، العراق }
\end{aligned}
$$

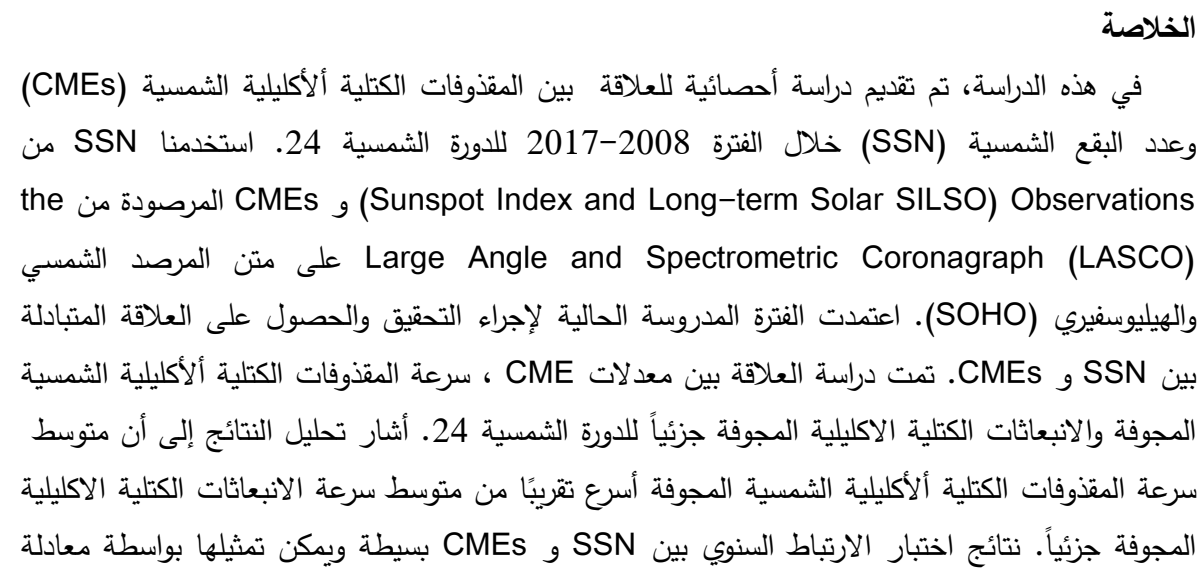

Email: zain1alhak1@scbaghdad.edu.iq 


$$
\begin{aligned}
& \text { انحدار خطية. و أخيراً تم عمل ملائمة كاوسية كدالة للزمن للمقارنة بين تصرف عدد SSN و مع مع } \\
& \text { السنوات وكانت النتائج ان مركز القمم تتطابق عند العام } 2014 .
\end{aligned}
$$

\section{Introduction}

Solar atmospheric phenomena gained increasing scientific interest during the past century mainly because of their expected effect on the climate of the Earth as well as radio communication stability. Besides that, these phenomena possess important clues about solar structure and its various internal reactions. This is why solar atmospheric physics became one of the richest and most dynamic environmental studies in modern astrophysics.

A key parameter in the solar atmospheric phenomena is the solar cycle which takes approximately 11 years on the average. During this time, the SSN starts from a minimal value and increases which marks the start of the solar cycle. Then, the SSN tends to reach a maximum, then decreases to a minimum again where a mark is given for the end of a solar cycle. The reason for this phenomenon is believed to be the continuous fluctuation of the solar magnetic field, and this belief is strongly supported by the observed fact that a sunspot maintains a strong magnetic field compared to that of the surface of the sun. The density and temperature of the outer layers of the sun get dynamically affected by the movement of the solar magnetic field emerging from the solar interior, therefore $\mathrm{s}$ sunspot holds remarkable and measurable parameters that were used to deduct the reason behind SSN cycle. These parameters recorded by many solar activities, such as the change of the SSN, frequency of CMEs occurrence, plasma temperature, and the speed of the solar wind [1].

Based on calculated SSN, which was considered to be the smallest number since the beginning of the space age, the solar cycle 24 was relatively weak but comparable longer than other near cycles [2]. It is thought to be due to the measured low polar magnetic field strength in solar cycle 23. According to Christensen-Lassen theory [3] that behavior of the cycle 24 will affect the cycle 25 by making the sun with a lesser irradiance. Besides that, numerous studies mentioned that the cycle 24's low activity may lead to a global minimum [4]. Since it is still not in reach to perform a comparable solar-like reaction in the laboratory, the basic physical process of the interaction between collective magnetic fields and solar surface's plasma still under debate [5].

The main role of the sun in space weather becomes prominent because of its day-night continuous cycle, and because the electromagnetic radiation it emits consists of two main components: semistable and horizontal radiation. The long-term variation in the energy of emitted solar photons can be associated with climate impacts, but the short-term variations in the solar energy, specially abrupt changes in the solar wind, will mainly affect the Earth ionosphere, which causes radio communication problems. According to Gopalswamy, turbulences in the solar wind consists of three components, CMEs, Solar energetic particles SEP and Solar flares [6].

CMEs cast a huge amount of masse, mainly energized plasma, into the interplanetary space. These massive emissions usually happen during the solar cycle maximum and travel into space with speeds that can reach up to $2000 \mathrm{~km} / \mathrm{s}$. These ejections are the main player in the space weather.

Space weather is a direct tool that reveals changes in the solar emission, such as energetic particle events and other phenomena that cause plasma heating and acceleration. Masses of solar plasma can also during solar flares. On the other hand, the Geomagnetic Storms (GMS) that take place near the Earth, are caused by the interactions by materials ejected from the Sun, mainly solar energetic particle (SEP) events, and the geomagnetic field of the Earth. That usually results in considerable disturbances in the Earth's magnetic field [7]. Thus it can be deduced that increasing the number of solar flares and CMEs will increase the chance that damage can occur in spacecrafts near Earth as well as global radio communication systems. Another possible danger of SEP is its possible effects on astronauts and airline travelers at high-altitudes and near polar regions [8].

The interaction of the geomagnetic field with the magnetic field occurs within CMEs and the surrounding background magnetised by the modulated solar wind. If the speed levels range from 400$2,500 \mathrm{~km} / \mathrm{sec}$, some 1-4 days are needed for CMEs to propagate from the Sun to the Earth, featuring a typical transmit time of 2-3 days. The correlations between the strength of CMEs and the magnitude of their impact in geospace continue to be investigated - both observationally and in numerical analyses [9]. The findings also indicated that the geomagnetic disturbances serve different space weather hazards, ranging from satellite systems to ground facilities like induction current, heating and expansion of polar upper atmosphere, drastic variation of radiation belt particle flux and the 
development of ionospheric storms; all these phenomena have been a subject of intensive space weather research and are worth being predicted accurately in practical space weather forecast [10].

A halo CME surrounds the occulting disk of the observation coronagraph in the plane's projection. Halo CMEs were first registered by Howard et al. (1982). Only a handful of CMEs were recorded by the Solwind coronagraph on board the P78-1 mission. Halo CMEs represent only about $3 \%$ of all CMEs. They also represent an energetic population because most CMEs that generate large SEPs and major geomagnetic storms are halos. A halo CME is usually created from close to the center of the disk, while about $10 \%$ are created close to the limb. In the halos of the limbs, the disorder that appears on the opposite side is likely to be unexpected [11].

On the photosphere the cooler and the active regions are Sunspots that releases a lower intensity of light in every directions. The sunspots advanced when concentrated magnetic field lines begin to emerge from the photosphere directed toward the solar corona. Because a condition called 'frozen-in' magnetic field the isolation of the plasma within these field lines stayed from the surrounding solar surface. The solar convection below the flux tube hampered by the orientation and force of the local magnetic field, resulting in fewer plasma particles within this region. Both pressure and temperature within the flux tube decrease while magnetic pressure keeps the flux tube from collapsing inward. Solar convection under the flux tube is impeded by the direction and force of the local magnetic field, which has reduced the number of plasma molecules in this region. Temperature and pressure drop in the flux tube and the magnetic pressure stops the breakdown into the flux tube [12].

CMEs are the most spectacular phenomenon of solar activity. They occur in regions of closed magnetic fields that overlie magnetic inversion lines [13]. A study on CME is an important topic that is related directly to the space environment [14]. The sunspot cycle is an important form of solar variability that indicates the extent of closed magnetic field structure on the sun and hence is important to the study of the origin of coronal mass ejections. The solar wind is a stream of charged particles released from the upper atmosphere of the Sun. This plasma consists of mostly electrons, protons and alpha particles. The magnetic field of the Sun, as well as different structures, waves and turbulent fluctuations on a wide range of scales are embedded within the solar wind. Webb \& Howard [15] studied CMEs from 1973 to 1989 concluding that CME occurrence frequency tends to follow the solar activity cycle in both amplitude and phase. Gopalswamy et al. (2009) [16] have also studied $\mathrm{CME}$ occurrence in relation to sunspot number and found that the correlation between them is quite weak during the maximum phase period of solar cycle as compared to that in both ascending as well as descending phase. Researchers have studied the solar cycle that ended in December 2008 which is known as solar cycle 23. This cycle was longer than normal. The present solar cycle 24 started in December 2008 and is expected to have a shorter time period.

In this paper we performed a statistical study for the relationship between CMEs and SSN from January 2008 to December 2017 for this cycle, depending on available observational data.

\section{MATERIALS AND METHODS}

\section{Data Selection}

In this research, the SSNs data were obtained from Sunspot Index and Long-term Solar Observations (SILSO), which is supported by the International Council for Science World Data System [17]. Meanwhile, the data on CMEs and halo CMEs were downloaded from the Coordinated Data Analysis Workshops (CDAW) catalog $[18,19]$ during the period from January 2008 to December 2017 through solar cycle 24.

\section{RESULTS AND DISCUSSION}

In this study, the statistical analysis was conducted to investigate the behaviors of SSN and CMEs and derive the mutual correlation between these parameters for the annual time during the period from January 2008 to December 2017 through solar cycle 24. Although the solar cycle number 24 started in December 2008, data were taken before that to increase the sample number which gives better statistics in the curve fitting. The speed of full halo CMEs and partial halo CMEs for solar cycle 24 were analyzed alongside the SSN and CME number. The angular width of $360^{\circ}$ was considered for full halo CMEs, while $121-359^{\circ}$ was considered for partial halo CMEs. Other sizes of angular widths could be narrow $\left(\sim 5\right.$ to $\left.120^{\circ}\right)$ or spike (less than $\sim 5^{\circ}$ ); and CMEs with these widths were not considered in the present research.

The size and speed of CMEs are important parameters to determine when attempting to predict if and when the CMEs will impact the Earth. These properties of CMEs can be estimated using 
observations from an instrument known as a coronagraph, such as the one in LASCO which blocks the bright light of the solar disk thus allowing the outer solar atmosphere (chromosphere and corona) to be observed.

A total of 6452.3 SSN and 15,946 CMEs were observed during the period from January 2008 to December 2017 through solar cycle 24. Out of these CMEs, 324 were full halo and 1040 were partial halo. This means that halo CMEs represented only about $2 \%$ of all CMEs, and partial halo represented only about $7 \%$ of all CMEs in this time period. Table-1 shows the values of the observed SSNs and CMEs for the selected period.

The number of CMEs that have a speed greater than or equal to $1000 \mathrm{~km} . \mathrm{s}^{-1}$ was 203 including 109 halo CME and 54 partial haloes during the period from January 2008 to December 2017 through solar cycle 24.

The total number of occurrence of CME in all months in 2014 were 2478 events, but when we sum the total sunspot number in all months in 2014, the result was found to be 1363.3.

The solar activity in 2014 was at its maximum in 2014. Therefore, it is higher than in other years in cycle 24.

The total sunspot number without means was calculated by summing the total sunspot number in ten years and the result was 6452.3, while the total number of occurrence of CMEs in ten years was 15,946 events (see Table-1). So, it is noted that the total sunspot number was smaller than the total number of occurrence of CME in cycle 24. It is important to know the exact relationship between sunspots number and CME and provide a tool to replace the study of one of these events.

The number of occurrence of CME slightly increases towards sunspots maxima and slightly decreases towards sunspots minima.

The highest percentage of halo CMEs were 3.9\%, 2.8\% recorded in 2012, 2014 respectively.

Table 1-Occurrence rates of SSNs andCMEs for 2008-2017 of solarcycle 24 [12-14]

\begin{tabular}{|c|c|c|c|c|c|c|c|}
\hline year & SSN & $\begin{array}{l}\text { \# no. of } \\
\text { all CME }\end{array}$ & $\begin{array}{c}\text { no. of CMEs } \\
\text { with } \\
\text { speed } \geq 1000 \\
\text { km.s }\end{array}$ & $\begin{array}{l}\text { Types of } \\
\text { CMEs }\end{array}$ & $\begin{array}{l}\text { No. of } \\
\text { events }\end{array}$ & $\begin{array}{c}\text { Average } \\
\text { speed } \\
\text { km.s }^{-1}\end{array}$ & $\begin{array}{l}\text { percentage of } \\
\text { halo CMEs \% }\end{array}$ \\
\hline \multirow{2}{*}{2008} & \multirow{2}{*}{49.8} & \multirow{2}{*}{863} & \multirow{2}{*}{1} & Halo & 1 & 143 & \multirow{2}{*}{0.1} \\
\hline & & & & Partial Halo & 12 & 389.3 & \\
\hline \multirow{2}{*}{2009} & \multirow{2}{*}{57} & \multirow{2}{*}{746} & \multirow{2}{*}{0} & Halo & 1 & 276 & \multirow{2}{*}{0.1} \\
\hline & & & & Partial Halo & 2 & 260.5 & \\
\hline \multirow{2}{*}{2010} & \multirow{2}{*}{298.8} & \multirow{2}{*}{1117} & \multirow{2}{*}{11} & Halo & 11 & 696.3 & \multirow{2}{*}{1.0} \\
\hline & & & & Partial Halo & 41 & 395.3 & \\
\hline \multirow{2}{*}{2011} & \multirow{2}{*}{969.1} & \multirow{2}{*}{1990} & \multirow{2}{*}{33} & Halo & 41 & 930.9 & \multirow{2}{*}{2.0} \\
\hline & & & & Partial Halo & 143 & 572.8 & \\
\hline \multirow{2}{*}{2012} & \multirow{2}{*}{1012.7} & \multirow{2}{*}{2177} & \multirow{2}{*}{43} & Halo & 84 & 949.0 & \multirow{2}{*}{3.9} \\
\hline & & & & Partial Halo & 189 & 549.1 & \\
\hline \multirow{2}{*}{2013} & \multirow{2}{*}{1124.5} & \multirow{2}{*}{2338} & \multirow{2}{*}{36} & Halo & 55 & 888.7 & \multirow{2}{*}{2.4} \\
\hline & & & & Partial Halo & 190 & 537.8 & \\
\hline \multirow{2}{*}{2014} & \multirow{2}{*}{1363.3} & \multirow{2}{*}{2478} & \multirow{2}{*}{42} & Halo & 69 & 908.5 & 0 \\
\hline & & & & Partial Halo & 265 & 502.4 & 2.8 \\
\hline 2015 & 8274 & 2058 & 24 & Halo & 41 & 873.5 & م \\
\hline 2015 & 851.4 & 2058 & 24 & Partial Halo & 128 & 528.5 & 2.0 \\
\hline & & $120 ?$ & 4 & Halo & 12 & 554.8 & 0 \\
\hline 2016 & 477.9 & 1393 & 4 & Partial Halo & 58 & 377.1 & 0.9 \\
\hline 2017 & 2610 & 786 & 0 & Halo & 9 & 1487.9 & 12 \\
\hline 2017 & 201.8 & 180 & 9 & Partial Halo & 12 & 291.7 & 1.3 \\
\hline total & 6452.3 & 15946 & 203 & & & & 2.0 \\
\hline
\end{tabular}




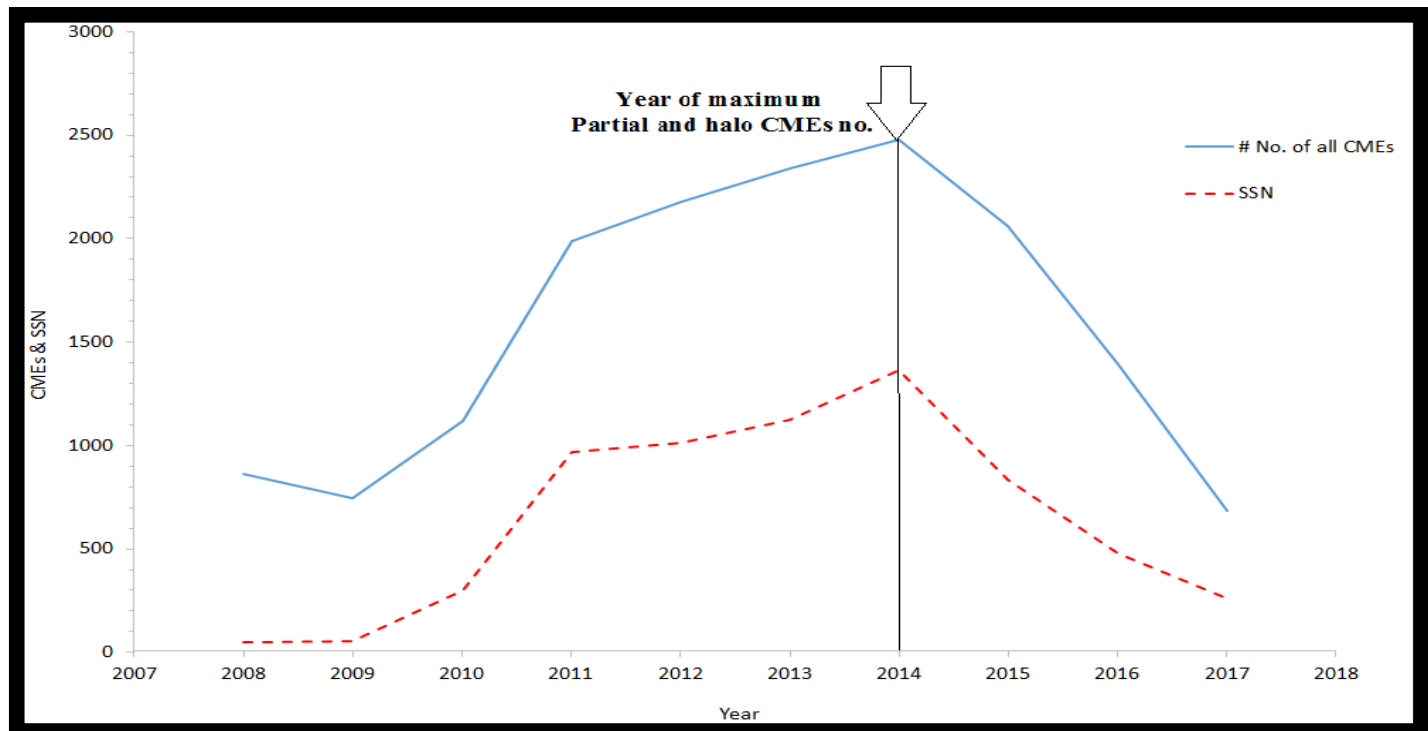

Figure1-Linear plot for the annual occurrence rates of CMEs and SSN for 2008-2017 of solar cycle 24.

Figure-1 shows the linear plot for the annual occurrence rate of CMEs and SSN for 2008-2017) of solar cycle 24. The occurrence rate of CMEs increases with the active region measured by the SSN. In this study, we find that the linear regression equation is $y=0.6607 \mathrm{x}-401.49$ and the correlation coefficient is $\mathrm{R}=0.975$, which measures the degree of a linear relation. A very good relationship rate with the positive slope is observed between the occurrence rate of CMEs and SSN, as shown in the correlation chart of Figure-2.

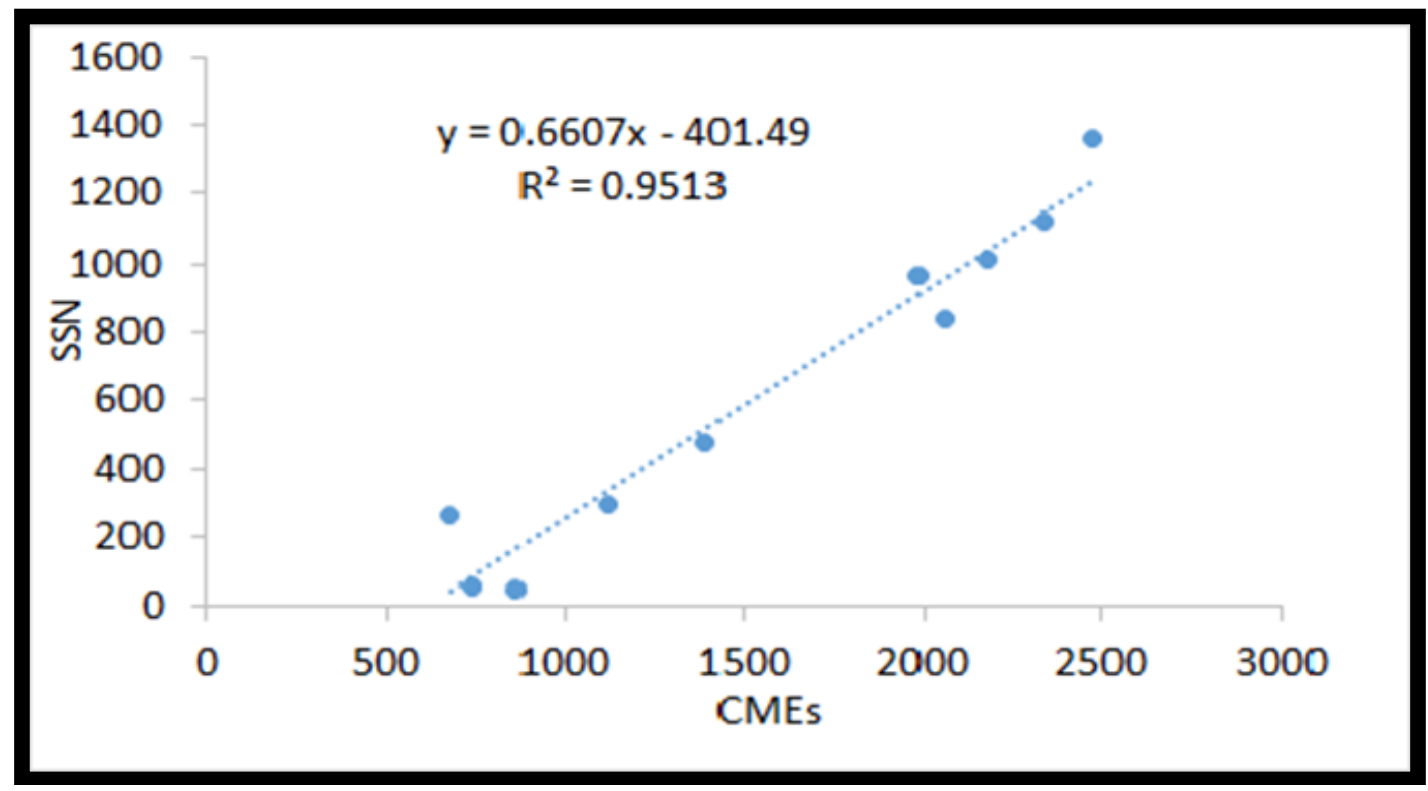

Figure 2-Correlation relationship between CMEs and SSN for 2008--2017 of solar cycle 24.

Moreover. Figure-3 illustrates that during solar cycle 24 the maximum SSNs and number of CMEs occurred in 2014, and the highest number of CME occurrences 43 with a speed greater than or equal to 1000 was in 2012, (Figure-3) these $43 \mathrm{CME}$ including 29 halos and 7 partial haloes. The maximum number of halo CMEs 84 with an average speed of 949 also occurred in 2012 (Figure-3), while the maximum number of partial halos were 265 with an average speed of 502.4 occurred in 2014 (Figure-4.) 


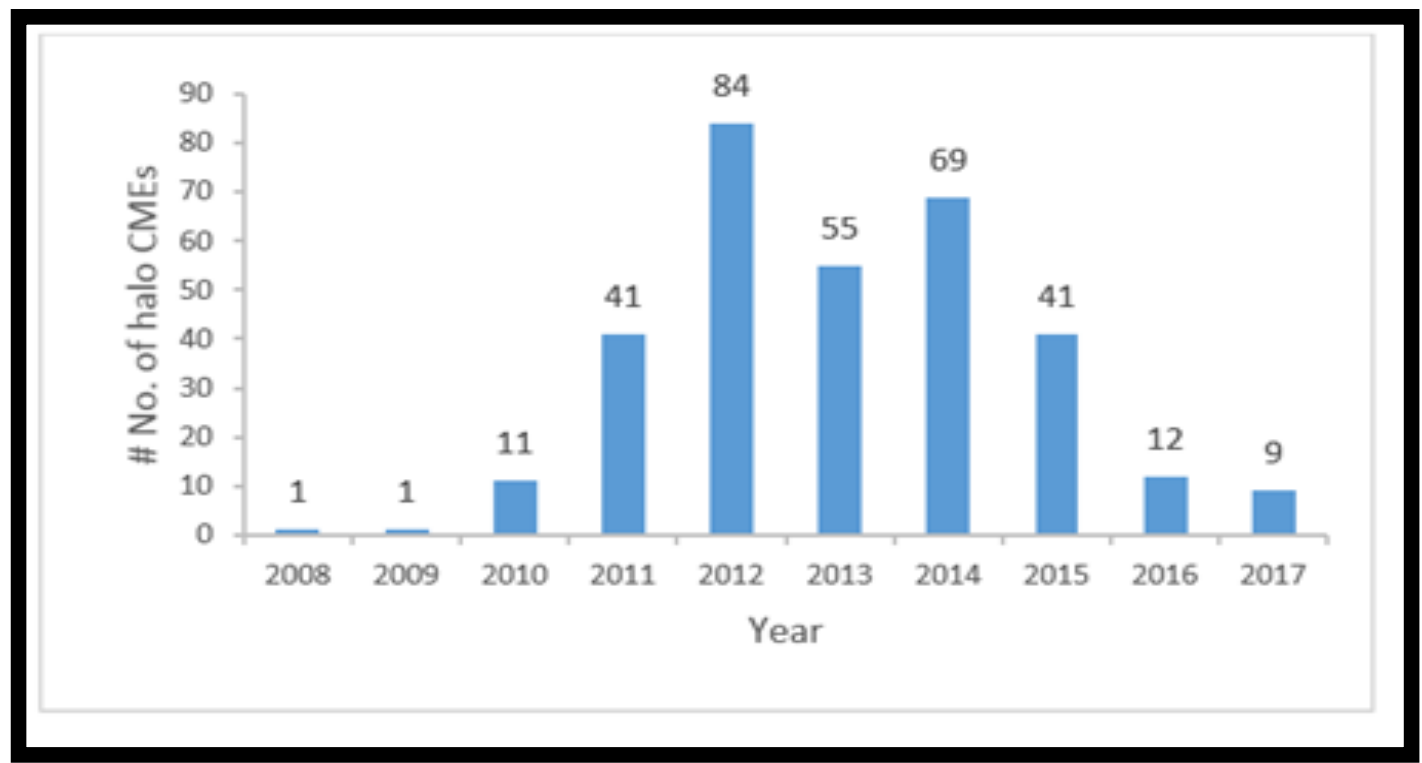

Figure 3-Number of halo CMEs per year for 2008-2017 of solar cycle 24.

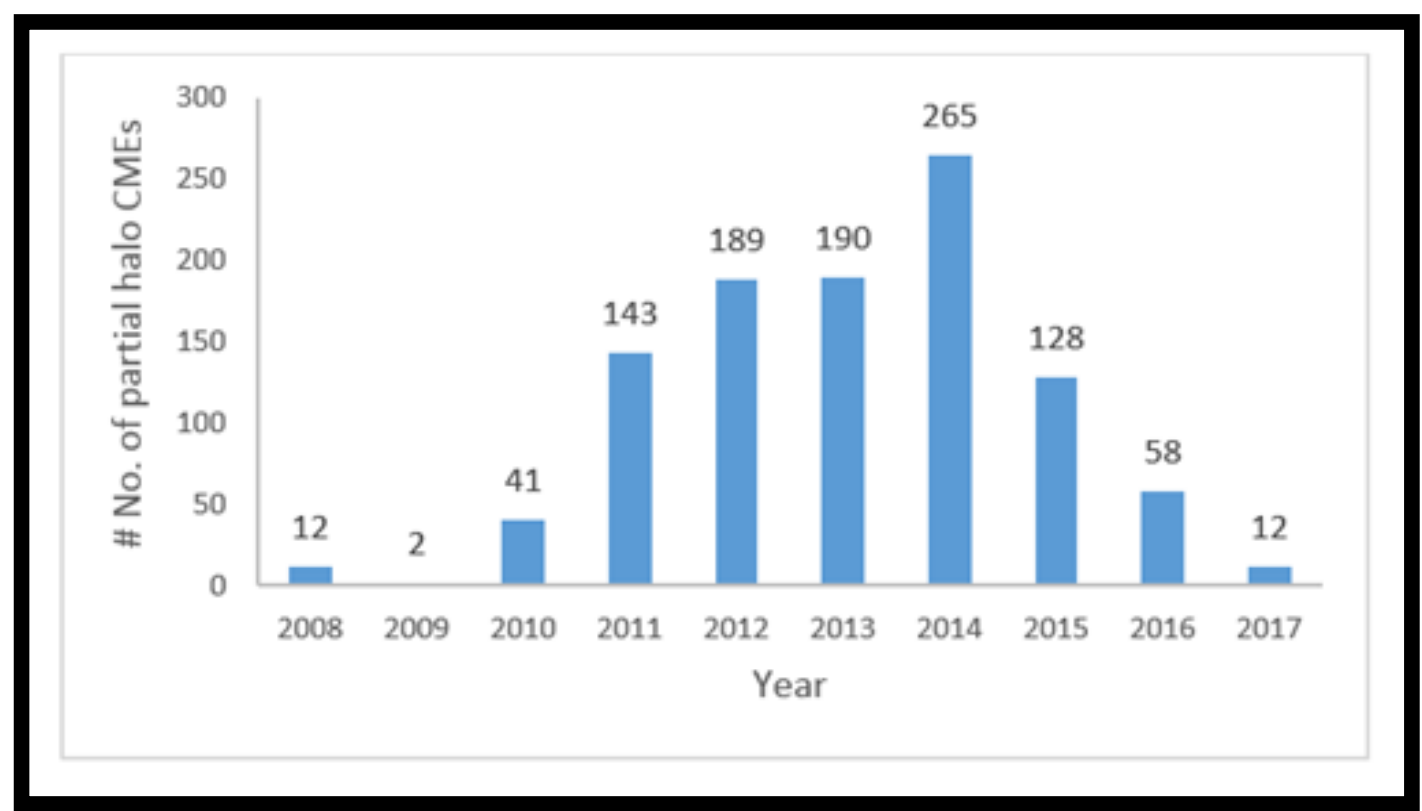

Figure 4-Number of partial halo CMEs per year for 2008-2017 of solar cycle 24.

Then, a more complicated curve-fitting procedure was performed for the data in Figure-1, where a Gaussian fit with single peak, i.e., with the relation: $f(x)=a \exp \left(-\frac{(x-b)^{2}}{c^{2}}\right)$ was made for both SSN and CMEs numbers against time. The Matlab fitting tool, cftool, was used for this task. Figure-5 shows the results of both fits. In Table-2 the statistics of both fits are given.

An important note that should be made here is, the Gaussian fit as a function of time can only be valid for comparison between CME and SSN number in this case, and the relations found from curve fitting should not be assumed as a general case because the Gaussian depends on time (in years) and these parameters has a cyclic role in the solar cycle. Nevertheless, a fair comparison can be deduced from this fitting as seen from Table-2.

It can be clearly seen that the Gaussian fit gave good approximation for both numbers of CME and SSN. Both fittings gave centers in 2014 with a relatively low standard deviation of less than 3\%. Furthermore, the constants that describe the behavior of the Gaussian, namely $b$ and $c$, were close in general, with again less than 3\% deviation. The constant that describes the height of Gaussian peak, the parameter a, was different since it describes the difference between the peaks of each curve. 
Table 2-Curve fitting results for SSN and CMEs with the Gaussian relation

\begin{tabular}{|c|c|c|}
\hline \multicolumn{3}{|l|}{$f(x)=a \exp \left(-\frac{(x-b)^{2}}{c^{2}}\right)$} \\
\hline & SSN & CME \\
\hline Mean Center & 2014 & 2014 \\
\hline Std div. on mean & $2.54 \%$ & $2.55 \%$ \\
\hline $\mathrm{a}$ & 1272 & 2502 \\
\hline $\mathrm{b}$ & -0.1349 & -0.132 \\
\hline $\mathrm{c}$ & 1.228 & 1.518 \\
\hline Goodness of fit $\left(\mathrm{R}^{2}\right)$ & $89.05 \%$ & $95.83 \%$ \\
\hline Adjusted $\mathrm{R}^{2}$ & $84.70 \%$ & $94.61 \%$ \\
\hline
\end{tabular}

The constants ratio that describes the behavior of the Gaussian for SSN and CME are:

$$
\begin{aligned}
& (a(S S N)) /(a(C M E))=0.50839 \\
& (b(S S N)) /(b(C M E))=1.02196 \\
& (c(S S N)) /(c(C M E))=0.80895
\end{aligned}
$$
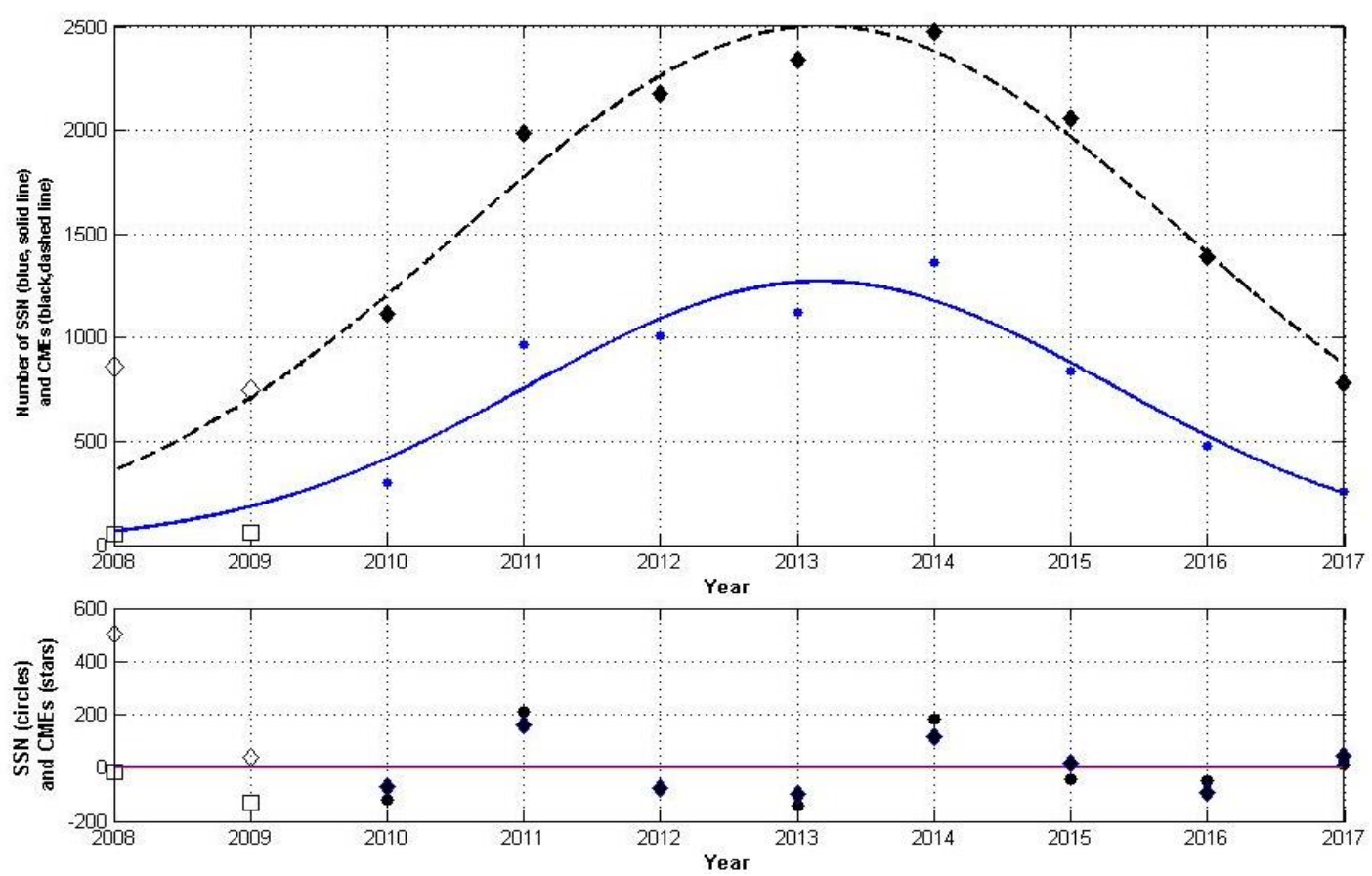

Figure 5-Upper plot: Gaussian curve-fitting for both of CMEs number (the black and dashed line, the black diamonds are the actual data) and SSN (the blue and solid line, the blue circles are the actual data) against time for the solar cycle 24. Lower plot: The residuals of both fits in the upper plot, where circles are for SSN and diamond shapes for CME. The vertical line in the lower plot is the zero-level. White circles and squares are excluded data from the fit.

The two points at the beginning of data (for years 2008 and 2009) were excluded while making the fitting in Figure-5. This is because if we included them the mean center of both CMEs and SSN numbers will be in the year 2013. It is commonly accepted that the maxima of both numbers occurred in 2014, and this result appeared from the statistics of the fitting when we excluded the points at years 2008 and 2009. Excluded points are shown as empty markers in both of the upper and lower parts of Figure-5.

\section{CONCLUSION}

1. The solar cycle 24 initially have activity less than cycle 23 . 
2 Emissions of CMEs can occur at any time during a solar activity but increase with solar activity and solar energy.

3. The present results indicated a high affiliation relation between CMEs and SSNs as expected since both generate from active regions of the sun.

4. When SSN increases, the number of CMEs slightly increases, and vice versa.

5. The maximum number of halo CMEs occurred in 2012 and 2014.

6. A good relationship with a positive slope exists between SSN and the occurrence rate of CMEs. The correlation coefficient between CMEs and SSN is 0.975.

7. The Gaussian fit gave a good approximation for both numbers of CME and SSN and results show that the center of the peaks agrees with 2014.

\section{Suggestions}

It is suggested to study previous solar cycles to find the relationship between (a (SSN))/ (a $(\mathrm{CME})),(\mathrm{b}(\mathrm{SSN})) /(\mathrm{b}(\mathrm{CME}))$ and $(\mathrm{c}(\mathrm{SSN})) /(\mathrm{c}(\mathrm{CME}))$ and compare them.

\section{REFERENCES}

1. Visakh kumar U.L, Bilin Susan Varghese, P.J Kurian .2017. Relation Between Solar Wind Parameters, Coronal Mass Ejections And Sunspot Numbers. International Journal of Engineering and Applied Sciences, 4(9): 68-70.

2. Scott W. McIntosh et al.2019. What the sudden death of solar cycles can tell us about the nature of the solar interior. astro-ph.SR, arXiv:1901.09083v1.

3. E.Friis-Christenscn; K.Lassen.1991. Length of the Solar Cycle: An Indicator of Solar Activity Closely Associated with Climate. JSTOR, 254(5032): 698-700.

4. N. Gopalswamyl, P. Ma.keltt, S. Akiyama, S. Yashiro, and N. Thakur. 2015. CMEs during the Two Activity Peaks in Cycle 24 and their Space Weather Consequences. Sun and geosphere, 10(2): $101-108$.

5. Bidhu S S , Iren Sobia A, Dickson Benjamin. 2017. Relation Between Solar Wind Parameters and Sunspot in Recent Solar Maxima. International Journal of Scientific Research in Science and Technology, 3 (7): 898-900.

6. Nat Gopalswamy.2007.Coronal mass ejections and space weather". Climateand WeatheroftheSun-EarthSystem: 77-120.

7. Guido, R.M.D. 2016. Coronal Mass Ejections during Geomagnetic Storms on Earth. International Journal of Astronomy. 5(2). DOI: 10.5923/j.astronomy/20160502.02.

8. Pulkkien, T. 2007. Space Weather: Terrestrial Perspective. Living Reviews in Solar Physics. 4(1).

9. Newell, P., Sotirelis, T., Liou, K., Meng, C.I., Rich, F.J. 2007. A Nearly universal solar haloes coupling function inferred from 10 magnetospheric state variables. JournalofGeophysicalResearch, 112,A21026DOI:10.1029/2006JA012015.

10. Miyake, W. and T. Nagatsuma.2012. On the predictive ability of geomagnetic disturbances from solar wind measurement at separated solar longitude. International Journal of Astronomy and Astrophysics, 2:63-73.

11. Bidhu S S, A Iren Sobia .2016. Coronal Mass Ejections in Solar Cycle 24, Journal of Applied Science and Engineering Methodologies, 2(3): 326-329.

12. Noor Alhuda Kamel, Ahmed Abdul-Razzaq Selman .2019.Automatic Detection of Sunspots Size and Activity using Matlab. Iraqi Journal of Science. 60(2):411-425.

13. Gosling, J.T. 1990.Coronal Mass Ejections and Magnetic Flux Rope in Interplanetary Space, AGU Monograph SeRies, 58: 343-364.

14. Fox, N.J., Peredo, M. and Thompson, B.J. 1998.Cradle to Grave Tracking of the January 6-11, 1997, Sun-Earth Connection Event, Geophysical Research Letters, 25(14): 2461-2464.

15. Webb, D.F. and Howard, R.A. 2012. The Solar Cycle Variation of Coronal Mass Ejections and the Solar Wind Mass Flux, Journal of Geophysical Research, 99(A3): 4201-4220.

16. Gopalswamy, N. 2010.Magnetic Coupling between the Interior and Atmosphere of the Sun ,Astrophysics and Space Science Proceedings, : 289-307.

17. https//sidc.oma.be/silso/home

18. https //cdaw.gsfc.nasa.gov/CME list/

19. https//cdaw.gsfc.nasa.gov/CME list/halo/halo html 